\title{
BODY COMPOSITION AT BIRTH; NORMATIVE VALUES
}

\author{
C.P. Hawkes ${ }^{1,2}$, J. O'B Hourihane ${ }^{1}$, L.C. Kenny ${ }^{3}$, A.D. Irvine ${ }^{4}$, M. Kiely ${ }^{5}$, D.M. Murray ${ }^{1}$ \\ ${ }^{1}$ Department of Paediatrics and Child Health, University College Cork, ${ }^{2}$ Department of Neonatology, Cork \\ University Maternity Hospital, ${ }^{3}$ Department of Obstetrics and Gynaecology, University College Cork, Cork, \\ ${ }^{4}$ National Children's Research Centre, Crumlin, Dublin, ${ }^{5}$ School of Food and Nutritional Sciences, \\ University College Cork, Cork, Ireland
}

Introduction: There is increasing evidence that in utero growth has immediate and long term health effects. Birthweight and length are used as surrogate measures of in utero growth. However, these measures poorly reflect neonatal adiposity and fat free mass. Air displacement plethysmography is validated for the measurement of body fat in the neonatal population. We aimed to establish normal reference values of body fat percentage $(\% \mathrm{BF})$ in infants in the first week of life.

Methods: As part of a large population based birth cohort study, fat mass, fat free mass and body fat percentage were measured in the first 4 days of of life, using air displacement whole body plethsymography. Infants were grouped into gestational age and sex categories.

Results: Of the 786 infants enrolled, 743 (94.5\%) infants had their body fat percentage (\%BF) checked within the first 4 days of life. Body fat percentage increased significantly with gestational age. Mean (SD) $\% \mathrm{BF}$ at $36-37^{+6}$ weeks' gestation $=8.9 \%(3.5)$, at $38-39^{+6}=10.3 \%(4)$, and at $40-41^{+6}=11.2(4.3)(\mathrm{p}<$ $0.001)$. Females had significantly increased mean (SD) $\% B F$ at $38-39^{+6}=11.1(3.9)$ vs $9.8(3.9)(p=0.012)$ and at $40-41^{+6}=12.4(4.4)$ vs $10(3.9)(\mathrm{p}<0.001)$. Sex and gestational specific centiles were calculated and a normative table was generated.

Conclusion: $\% \mathrm{BF}$ at birth is influenced by gestational age and sex. We have generated accurate $\% \mathrm{BF}$ centiles from a large population based cohort. 